\title{
Speed of Light as an Emergent Property of the Fabric
}

\author{
Dirk J. Pons ${ }^{1}$, Arion D. Pons ${ }^{2} \&$ Aiden J. Pons ${ }^{3}$ \\ ${ }^{1}$ Department of Mechanical Engineering, University of Canterbury, New Zealand \\ ${ }^{2}$ University of Canterbury, Christchurch, New Zealand \\ ${ }^{3}$ Rangiora New Life School, Rangiora, New Zealand \\ Correspondence: Dirk Pons, Department of Mechanical Engineering, University of Canterbury, Private Bag 4800, \\ Christchurch 8020, New Zealand. Tel: 64-3364-2987. Email: dirk.pons@canterbury.ac.nz
}

Received: April 15, $2016 \quad$ Accepted: May 4, $2016 \quad$ Online Published: May 24, 2016
doi:10.5539/apr.v8n3p111
URL: http://dx.doi.org/10.5539/apr.v8n3p111

\begin{abstract}
Problem- The theory of Relativity is premised on the constancy of the speed of light (c) in-vacuo. While no empirical evidence convincingly shows the speed to be variable, nonetheless from a theoretical perspective the invariance is an assumption. Need- It is possible that the evidence could be explained by a different theory. Approach- A non-local hidden-variable (NLHV) solution, the Cordus particule theory, is applied to identify the causes of variability in the fabric density, and then show how this affects the speed of light. Findings- Under these assumptions the speed of light is variable (VSL), being inversely proportional to fabric density. This is because the discrete fields of the photon interact dynamically with the fabric and therefore consume frequency cycles of the photon. The fabric arises from aggregation of fields from particles, which in turn depends on the proximity and spatial distribution of matter. Results disfavour the universal applicability of the cosmological principle of homogeneity and isotropy of the universe. Originality- The work proposes causal mechanisms for VSL, which have otherwise been challenging to ascertain. Uniquely, this theory identifies fabric density as the dependent variable. In contrast, other VSL models propose that c varies with time or some geometric-like scale, but struggle to provide plausible reasons for that dependency. This theory also offers a conceptually simply way to reconcile the refraction of light in both gravitational situations and optical materials.
\end{abstract}

Keywords: non-local hidden-variable solution, variable speed of light, cosmological principle

\section{Introduction}

The constancy of the speed of light in the vacuum was the central insight in Einstein's work on general and special relativity (Einstein, 1920), and forms the foundation of cosmological theory. There is no empirical evidence that convincingly shows the speed of light to be variable in-vacuo. Nonetheless, from a theoretical perspective the invariance is an assumption, and it is possible that the empirical evidence could be explained by a different theory. In this paper we develop such a theory.

We show that a variable speed of light (VSL) is a logical outcome for the non-local hidden-variable (NLHV) solution of the Cordus theory. Postulating a VSL theory is not new, but other VSL theories are focussed on cosmological physics, whereas this originates in fundamental physics.

\section{Background}

\subsection{Invariance of the Speed of Light}

The aberration of light, whereby the location of stars appeared to change depending on the velocity of Earth in its orbit, was an early indication that light had a finite speed (Bradley, 1729). In the late 1800s the propagation of light was believed to be through a medium called the luminiferous ether. One of these theories, the undulatory theory of de Broglie, Fresnel, Schrödinger and others (de Broglie, 1925; Schrödinger, 1926), proposed that the ether was generally at rest, except in transparent bodies where it caused refraction. The Michelson-Morley experiment (Michelson \& Morley, 1887) directly tested the idea of Earth moving through a stationary ether, by looking for differences in the speed of light in orthogonal directions, and found no evidence to support such a theory.

Einstein subsequently built special relativity (SR) on the radical premise that the speed of light is the same for all inertial reference frames (Einstein, 1920). Specifically, that the speed of light is invariant under any velocity or orientation of the inertial frame of observation (Lorentz transformation). Yet the constancy of the speed of light is 
nonetheless an assumption. Einstein guessed rather than proved it, and designed it into special relativity. The invariance is therefore a premise of SR, rather than a proof thereof. However what is more convincing is that SR predicts time dilation and the relativity of simultaneity, and these do appear to be real. Furthermore, many subsequent measurements have supported the constancy of the speed of light. The most recent (Herrmann et al., 2009) set a lower limit on any local anisotropy $\Delta c$ of the speed of light $c$ as $\Delta c / c \sim 1 \times 10^{-17}$, this experiment being conducted over one year, using a block of fused silica.

\subsection{Variable Speed of Light Theories}

The constancy of the in-vacuo speed of light, at least on Earth, is well-established, as are the effects of SR. Nonetheless there has been ongoing interest in whether the speed of light really is invariant (Albrecht \& Magueijo, 1999; Barrow \& Magueijo, 1999; Bassett, Liberati, Molina-Paris, \& Visser, 2000; Clayton \& Moffat, 2003; J. Magueijo, 2000; Magueijo, 2001, 2003, 2008; Moffat, 2005; Singha \& Debnath, 2007; Sisterna, 2011; Szydlowski \& Krawiec, 2003; Unzicker, 2009). The primary purpose of these endeavours has been to explore for new physics at deeper levels, with a particular interest in quantum-gravity. One approach has been to look for violation of Lorentz invariance in other settings (João Magueijo, 2000). For example, it may be that the invariance of $c$ breaks down at very small scales, or for photons of different energy. However such searches have been unsuccessful (Abdo et al., 2009).

Another approach is cosmological. If the speed of light was to be variable, it could solve certain problems. Specifically, it has been proposed that the horizon, inflation and flatness problems can be resolved if there were a faster $c$ in the early universe, i.e. a time-varying speed of light (Sisterna, 2011). There are several other applications of variable speed of light (VSL) theories (Racker, Sisterna, \& Vucetich, 2009; Sisterna, 2011) including branes (Youm, 2001) and particle creation (Harko \& Mak, 1999). Another focus has been to use VSL to explain the Pioneer anomaly (Berman, 2011), e.g. using power law fitting (Shojaie, 2012).

In all these theories the difficulty is providing reasons for why $c$ should vary with time or scale. They require the speed of light to be different at genesis, and then somehow change slowly or suddenly switch over at some time or event, for reasons unknown. None of these theories describe why this should be, nor do they propose underlying mechanics. This is ontologically problematic. Existing VSL theories cannot show a causal connection with existing physics, and therefore are not widely accepted.

\section{Purpose and Approach}

The purpose of this work was not initially to develop a VSL theory, though that is one of the outcomes achieved, but rather to identify whether there was a mechanism within the Cordus theory for $\mathrm{c}$ to have the value it does.

The Cordus theory is a specific type of NLHV solution (D. J. Pons, A. D. Pons, A. M. Pons, \& A. J. Pons, 2012). Under this framework the vacuum is not entirely empty but comprises a fabric of discrete fields emitted by all particles (D. J. Pons \& A. D. Pons, 2013). The approach taken here is to identify the causes of variability in the fabric density, and then show how this affects the speed of light.

\section{Results}

\subsection{Fabric of the Vacuum}

\subsubsection{Structure of the Cordus Particule}

The starting premise of the Cordus conjecture is that particles are not zero-dimensional (0D) points but rather have an internal structure. This structure is proposed to comprise two reactive ends, which are a small finite distance apart (span), and each behave like a particle in their interaction with the external environment (Pons et al., 2012), see Figure 1. A fibril joins the reactive ends and is a persistent and dynamic structure but does not interact with matter. It provides instantaneous connectivity and synchronicity between the two reactive ends. This structure was first used to explain wave-particle duality in the double-slit device (Pons et al., 2012), and has subsequently been applied to diverse other phenomena such as nuclide stability/instability (D. J. Pons, A. D. Pons, \& A. J. Pons, 2013a, 2015c) and asymmetrical baryogenesis (D. J. Pons, A. D. Pons, \& A. J. Pons, 2014a).

This is a non-local solution: the particule is affected by more than the fields at its nominal centre point. Each reactive end of the particle is energised in turn at the de Broglie frequency of that particle, which is dependent on its energy. The reactive ends are energised together for the photon, and in turn for matter particules (D. J. Pons, 2015). The span of massy particles shortens as the frequency increases, i.e. greater internal energy is associated with faster re-energisation sequence, hence also faster emission of discrete force and thus greater mass. 


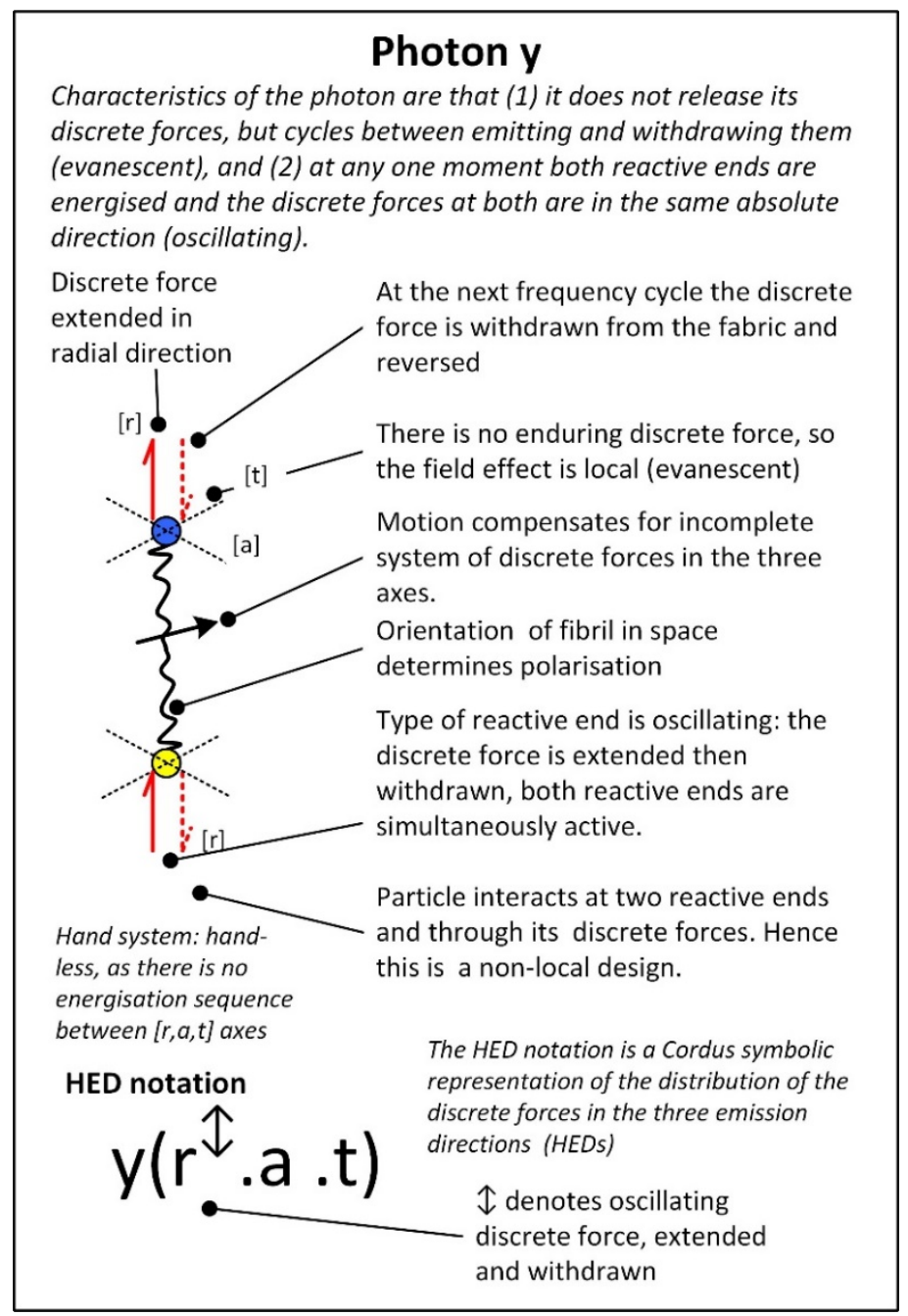

Figure 1. The structure of the photon as proposed by the Cordus theory. The particule has an internal structure comprising two reactive ends that energise together (whereas massy particules energise in sequence), and emit discrete forces into the fabric in the process. The emission is reversed at the next frequency cycle. Hence the energy is extracted from the fabric at one reactive end and instantaneously emitted at the other, and then this cycle is reversed. In contrast massy particules release their discrete forces, and this makes up a fabric of discrete forces in the vacuum. Image reproduced from (Pons, 2015) by permission of the author

The Cordus theory proposes the following lemma:

Each interaction of a photon with the fabric requires a frequency cycle. (Lemma 1)

Consequently the de Broglie frequency of a particule is inversely proportional to fabric density.

\subsubsection{The Nature of Forces and Fields}

The theory proposes that each matter particle emits discrete forces at each energisation, and these are propagated out into the external environment surrounding the particle. The particle continues to emit more discrete forces each time its reactive ends energise. This sequence of emissions makes up a flux tube of discrete forces that are propagated into the external environment. Furthermore the discrete forces are not consumed by interactions with other particles, but instead continue to travel away from the particule that emitted them. The aggregation of many such discrete forces from many neighbouring particules creates the electro-magnetic-gravitational (EMG) fields.

A physical explanation then arises for fields, namely that the direct lineal effect of the discrete force provides the electrostatic interaction, the bending of the flux line carries magnetism, and the torsion provides the gravitation interaction. These are all interactions of discoherent matter, whereas the strong force is predicted to be only applicable to coherent assemblies (D. J. Pons, A. D. Pons, \& A. J. Pons, 2013b). 


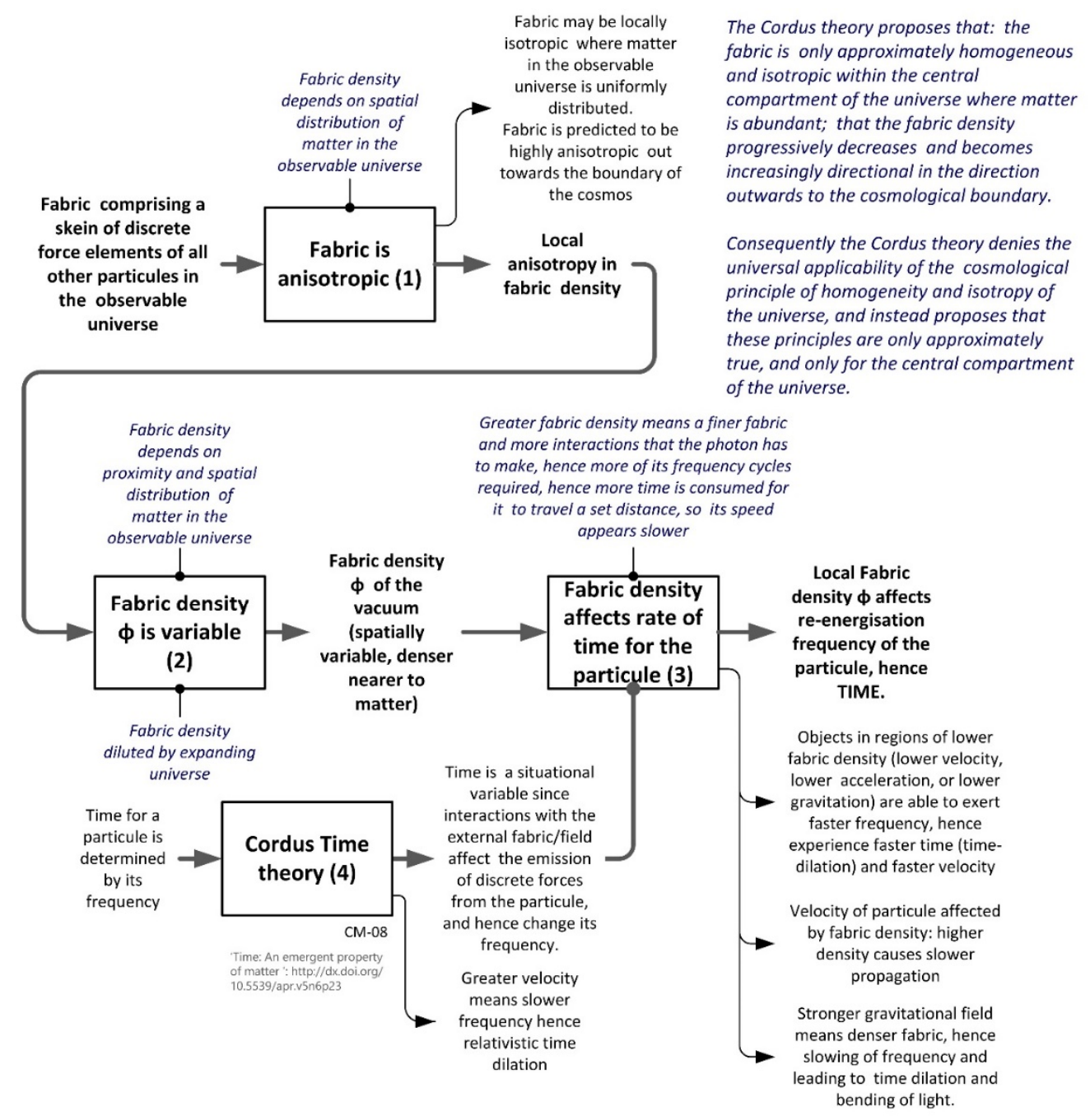

\section{CM-07-03-08 Variable Fabric density}

Figure 2. The fabric is proposed to have a variable density, due to the spatial distribution of matter within the universe. Consequently there are two effects to consider, the anisotropy and density of the fabric. It is the density

effects that are of particularly interest here, because of the implications for the speed of light. This diagram represents the proposed relationships of causality and the logical structure of the argument, and uses the integration definition zero (IDEF0) (FIPS, 1993) systems engineering notation

\subsubsection{Fabric}

The overall result is that the space in and between matter particules is filled with a fabric of discrete forces (Pons \& Pons, 2013). The fabric therefore corresponds in the first instance to the gross (rather than net) electrostatic field, more specifically to the local sum of the absolute values of the individual electric fields from negative and positive charges. Since matter in large quantities is most commonly neutral in charge, the net electrostatic field can be negligible small, but even in such situations the gross field may be large. It is therefore more helpful to associate the fabric with the gravitational field, which takes one sign only, and is more apparent at cosmological scales. In both cases the form of the dependency is the same, being $1 / \mathrm{r}^{2}$.

Thus in the Cordus theory the fabric comprises the moving discrete forces generated by matter particules within the observable universe (D. J. Pons \& A. D. Pons, 2013). It is relevant to note that this fabric can be relativistic 'in that for an isotropic fabric the propagation speed of the photon is the same in any direction, regardless of the motion of the body emitting the photon' (D. J. Pons \& A. D. Pons, 2013). Thus relativity can be recovered, at least in special inertial frames of reference where the fabric happens to be isotropic. 
The fabric also distinguishes the vacuum within the universe from the void before genesis, and is the reason for the existence of electrical and magnetic constants of the vacuum. The Cordus theory further requires that the density of the fabric $\varphi$ determines those constants and gives the speed of light a particular value.

\subsection{Variable Fabric Density}

The logical consequence of this hypothesis is that the fabric density of the vacuum is spatially variable, being determined by the proximity and local spatial distribution of matter, and in this regard is no different to the variability of EMG field strength. Hence also the fabric density is constrained by the observable universe. Thus matter that is too far away might be unable to send its discrete forces to a specific location of interest, and therefore does not contribute to the fabric density at that point. The complementary principle is that the fabric density is diluted by an expanding universe. These principles are summarised in the systems notation of Figure 2.

Furthermore, this means that the fabric will be anisotropic, since it depends on the spatial distribution of matter. It may be locally isotropic where the matter is uniformly distributed. However the theory predicts that the fabric will be highly anisotropic out towards the boundary of the cosmos (D. J. Pons \& A. D. Pons, 2013).

Thus it is proposed (D. J. Pons \& A. D. Pons, 2013):

(1) That the fabric is only approximately homogeneous and isotropic within the central compartment of the universe where matter is abundant;

(2) That the fabric density progressively decreases and becomes increasingly directional nearer the cosmological boundary.

Consequently the Cordus theory denies the universal applicability of the cosmological principle of homogeneity and isotropy of the universe, and instead proposes that these principles are only approximately true. Nonetheless, for privileged situations within the universe, such as inside galaxies or far from any galaxy, the night sky is approximately homogenous, and therefore the fabric density is also approximately isotropic and apparently constant in such situations.

Having proposed a means whereby the fabric may be inhomogeneous, we now consider the implications for the speed of light.

\subsection{Dependency of Speed of Light on Fabric Density}

\subsubsection{Propagation of the Photon in the Fabric of the Vacuum}

The Cordus theory proposes that the photon is different to other massy particules in that its discrete forces are emitted and withdrawn, as opposed to being released (Pons, 2015). The discrete forces of the photon can alternatively be considered to involve energy that is borrowed from the fabric. Consequently the photon can also be considered a type of electromagnetic transient within the fabric. This explains why many photons can share the same space: one photon imposes a distortion on the discrete forces of the fabric, and the distortions of multiple co-located photons are cumulative. This is consistent with electromagnetic (EM) wave theory, though the Cordus theory proposes a dipole arrangement rather than a point, and discrete forces rather than continuous EM fields.

Thus we propose that the photon interacts dynamically with the fabric. Each interaction requires a frequency cycle of the photon, since the photon's discrete forces are only generated when it re-energises. Hence frequency cycles of the photon are consumed with fabric interactions. This implies that denser fabric requires more frequency cycles of the photon per distance traversed (and less dense fabric requires fewer photon frequency cycles).

Complementary to this is the Cordus theory for time (D. J. Pons, A. D. Pons, \& A. J. Pons, 2013c), which proposes that time at the fundamental level consists of the frequency oscillations of the particules concerned. Thus time at the fundamental level is locally generated and an emergent property of matter, rather than being universal or being a dimension of space-time. Nonetheless the space-time concept can be recovered for the macroscopic level. This is because a disturbance in one body (e.g. its movement in space) is communicated to other bodies via photons, massy particules, or discrete fields, Thus time at the macroscopic level is also universal and relative, which is consistent with the perspective of general relativity.

Consequently the Cordus theory predicts that the time taken for the photon to traverse space depends on the fabric density. This is because greater fabric density means more interactions that the photon has to make, hence more of its frequency cycles are required, hence time runs slower for the photon relative to a frame of reference with sparser fabric. Thus we propose a time dilation effect whereby the photon takes longer time to cross a unit distance when in a fabric that is denser than the Observer's frame of reference. This conclusion also relies on a lemma: 
That fundamental spatial geometry is invariant, such that it is meaningful to consider a unit distance being the same whatever the fabric density.

This is consistent with the Lorentz length contraction being a kinematic effect associated with the time-dilation and the relativity of simultaneity, rather than being a static effect.

The outcome is that the Cordus theory predicts that the speed of light depends on the fabric density, which in turn depends on the proximity and spatial distribution of matter. Higher (lower) fabric density is expected to cause slower (faster) photon propagation. The system model for this is shown in Figure 3.

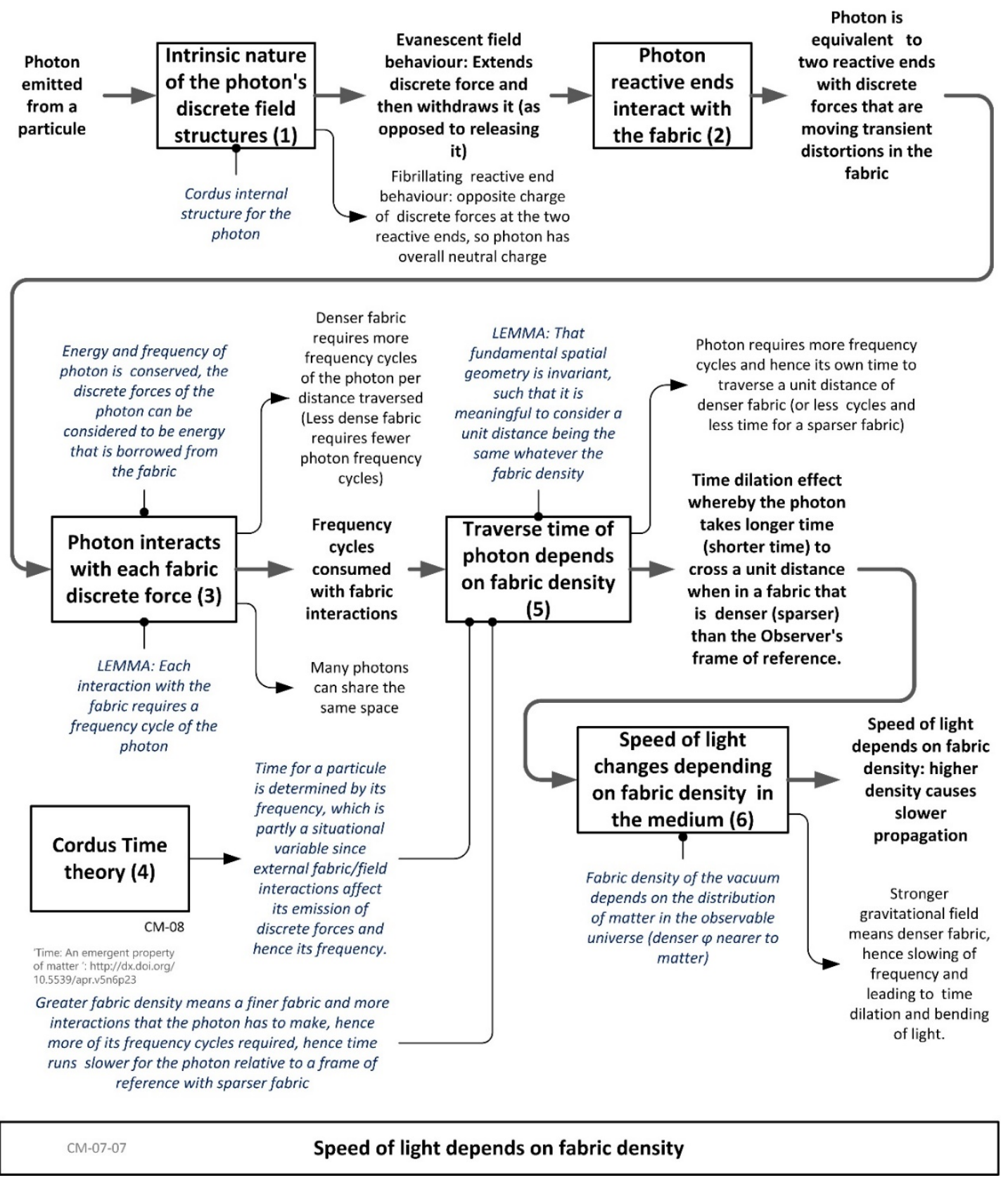

Figure 3. The Cordus theory predicts that the speed of light depends on the fabric density, which in turn depends on the proximity and spatial distribution of matter. Higher (lower) fabric density is expected to cause slower (faster) photon propagation 


\subsubsection{Fabric Density Transformations for Speed of Light In-Vacuo}

We introduce the new term situation to refer to an inertial frame of reference with a specific fabric density $\varphi$. Thus in this theory the local fabric density is an important variable along with the motion of the observer. In contrast relativity is only concerned with the latter.

In the general case the particule $B$ with velocity $\mathrm{v}_{\mathrm{B} 1}$ starts in situation 1 with fabric density $\varphi_{1}$ and frequency $\mathrm{f}_{\mathrm{B} 1}$. It subsequently moves into situation 2 of different fabric density $\varphi_{2}$ where its velocity becomes $v_{B 2}$ and its frequency $\mathrm{f}_{\mathrm{B} 2}$ as measured in situation 2. The speed of light is the saturated speed of propagation of discrete forces. In turn that is affected inversely by the fabric density. Hence the speed of light in situation 2 becomes:

$$
c_{2}=c_{1} \frac{\phi_{1}}{\phi_{2}} \text { or } c_{2} \phi_{2}=c_{1} \phi_{1}
$$

For example, if the Observer travelling with particule B moves into a situation $\varphi_{2}$ of lower fabric density, then the local speed of light $c_{2}$ increases, though it remains relativistic (is not affected by the velocity of the emitting particule) and is homogenous within that situation (providing there is no gradient to the fabric density). Consequently the Cordus theory is a variable speed of light (VSL) theory, in addition to being a non-local hidden-variable theory with discrete field emissions.

\section{Discussion}

\subsection{Outcomes}

This theory proposes that the speed of light is dependent on fabric density. In turn fabric density depends on the spatial distribution of matter in the local situation and the wider universe. This is a novel contribution to the VSL field, because (a) it proposes an underlying physical mechanism for the speed of light to be variable, which is otherwise exceedingly problematic, (b) it predicts a specific relationship between speed of light and fabric density, and this may be testable and falsifiable, (c) it recovers the Einsteinian assumption of the invariance of the speed of light, as a special case of isotropic fabric density, (d) it accommodates the relativity of light emissions, i.e. that the emitted speed of light is independent of the velocity of the emitting body. Thus the theory is not hostile to general and special relativity, but asserts that those theories are restricted to special situations where fabric density is constant.

\subsection{Applications}

\subsubsection{Why does the In-Vacuo Speed of Light have the Value it Does?}

The speed of light we observe is merely the value that occurs in our vicinity due to the background fabric density in this part of the universe. In turn, that is determined by the distribution of matter in the Milky Way plus contributions from the rest of the universe. Those other contributions are predicted to decrease as the universe expands, hence increasing the local speed of light as the universe evolves.

\subsubsection{How does Gravitational Bending of Light Occur?}

The gravitational field around a star creates a radial gradient in the fabric density. A photon with a Cordus particule structure has two reactive ends, and the discrete forces of these recruit a volume of space around each end. Where the density is higher, the recruitment process takes more time, i.e. there is a tendency for that reactive end to energise at a slower frequency. However the fibril mechanics of the particule does not permit different frequencies of the two reactive ends, as this would desynchronise the assembly and cause instability. Consequently the reactive end that experiences the greater fabric density, the one more medial to the star, recruits a smaller volume of space in the time available and thus moves forward a smaller increment. Hence the velocity of that reactive end is slower, and the locus of the particule is bent. This theory also predicts that the effect should depend on initial photon polarisation.

\subsubsection{Refraction at Transparent Bodies}

A body of matter, e.g. a glass prism, locally densifies the fabric density. The incident photon experiences a change in fabric density when it encounters the optical interface between the first medium (e.g. air) and the body of matter. That reactive end closest to the situation of higher fabric density will be retarded in its motion, as per the gravitational explanation above, and the locus with therefore bend. This is also consistent with the observation that transparent materials of higher density have higher refractive indices. Elsewhere the Cordus theory recovers the quantitative formalisms for reflection and refraction (Pons et al., 2012).

\subsection{Implications}

The outcome is that the Cordus theory rejects the concept of the invariance of the in-vacuo speed of light. Consequently this theory is contrary to special and general relativity. Nonetheless the theory accepts relativity as valid concept where the fabric density is sufficiently constant. Consequently the Cordus theory denies the 
universal applicability of the cosmological principle of homogeneity and isotropy of the universe, and instead proposes that these principles are only approximately true. The Cordus theory accepts the existing mathematical formulism of relativity and quantum mechanics as useful approximations in certain well-defined situations.

There are a number of implications of this theory. We have anticipated a mechanism which causes the speed of light to be situationally specific. This is conceptually consistent with gravitational bending of light and with relativity as a special case for situations where fabric density is constant. But what of situations where the fabric density is anisotropic? A variable speed of light has wide-ranging implications for theory development, since all of relativity is premised on its constancy. One example would be the black hole event horizon. The Cordus theory proposes that the speed of light is inversely proportional to fabric density, which in turn depends on the gravitational field strength. Consequently the speed of light slows down in a stronger gravitational field. This means that the light attempting to escape a black hole would be slowed, not stopped, thus there will be no event horizon. Instead the emitted light will be increasingly red-shifted and delayed in its escape. Furthermore the Cordus theory predicts that matter particles occupy a volume (the space between their reactive ends), and consequently there is no singularity at the centre of the black hole. These predictions have further implications for black hole cosmology. Obviously the Cordus theory is conjectural at this stage, so these implications are not definite. Nonetheless there are potentially profound implications if the theory were to be valid, and even if it were not there is still value in providing candidate new solutions to old unsolved-problems.

\subsection{Limitations}

While the implications may be profound, the concept still has many limitations. The work presented here is primarily of a theory-building type, and thus conceptual in nature. Consequently the mathematical formalism is not explicitly developed, and this is left for future work. It is also an exotic theory, on several counts: it proposes structure at the sub-particle level; it is non-local; it includes the concept of a fabric; it proposes that time is not a dimension but a property of matter; it is a variable speed of light theory; it denies the universal applicability of the cosmological principle of homogeneity and isotropy of the universe. It is thus appropriate to be cautious about the validity of the theory, and to consider it a candidate new physics rather than a proven fact.

\subsection{Implications for Future Research}

If this theory is true then the many applications in cosmology that assume invariance of $c$ would need to be revisited. The Cordus theory started as a NLHV theory at the particle level, as evidenced by the multiple papers on particle related topics such as nuclear structure and decay processes, e.g. (D. J. Pons, A. D. Pons, \& A. J. Pons, 2014b, 2015a, 2015b; Pons et al., 2015c; D. J. Pons, A. D. Pons, \& A. J. Pons, 2015d). However the same principles have also been found to be applicable at the cosmological scale. Specifically, the theory provides candidate solutions for a genesis route from pair production (Pons et al., 2015d) through to asymmetrical genesis (Pons et al., 2014a). The asymmetrical genesis theory is a novel proposition in its own, in that it proposes a production route that has not previously been considered and which is falsifiable. It also solves both the baryogenesis and leptogenesis problems simultaneously, which is otherwise difficult to accomplish. Other aspects of the theory extend to the horizon problem (D. J. Pons \& A. D. Pons, 2013) and to a novel formulation of the origins and arrow of time (Pons et al., 2013c). It is interesting that all these solutions do not conflict with either quantum mechanics or relativity. Instead those other theories become approximations of the deeper mechanics conceptualised by the Cordus theory. To the extent that the Cordus concept may be valid, it is therefore worth further developing and testing the theory for its potential to provide a deeper unification. Future research work streams could be directed at transforming the conceptual representation into mathematical formalism, which would be amenable to further investigation. There is also other conceptual work that might be undertaken, for example to explore gravitational-inertial mass equivalence, alternative explanations of dark matter, dark energy, and inflation.

\section{Conclusion}

The Cordus theory rejects the concept of the invariance of the in-vacuo speed of light, and instead proposes that the speed depends on the fabric density. The theory also provides a description of the composition of that fabric. This is in terms of an aggregation of discrete forces from many neighbouring particules to create an electro-magneto-gravitational (EMG) fabric.

There are several key concepts that differentiate this Cordus theory from other VSL theories. The first is the provision of a NLHV structure, the discrete force concept, and the idea that the fabric density at a location is variable being determined by the proximity and local spatial distribution of matter. The second is the idea that the discrete fields of the photon interact dynamically with each discrete force of the fabric and therefore consume frequency cycles of the photon. The third is that frequency cycles correspond to elapsed time, and therefore the 
traverse time of the photon depends on the fabric density. Thus we are proposing a time dilation effect whereby the photon takes longer time (shorter time) to cross a unit distance when in a fabric that is denser (sparser) than the Observer's frame of reference.

This work makes several original contributions. The first is to provide a variable speed of light theory with a systematic conceptual grounding. The argument starts with reasonable assumptions from a NLHV design, and builds an explanation for VSL behaviour. The originality is doing this from the fundamental level upwards. In contrast existing VSL theories are posterior constructs, which do not explain why c should vary.

A second contribution is the specific identification of fabric density as the dependent variable. In contrast, other VSL models propose that $c$ varies with time or some geometric-like scale, and then struggle to provide naturally plausible reasons why $c$ should depend on that variable. The Cordus theory is unique in proposing fabric density as the variable. This also means that compared to other VSL theories, the Cordus theory does not require an abrupt switch in $c$ during cosmological epochs.

A third contribution is that this theory offers a conceptually simply way to reconcile the refraction of light in $A L L$ settings, vacuum and optical materials. Optical theory already accepts that the speed of light is variable in any matter-based medium. Furthermore it is a general principle of optics that the refractive index increases with the mass density of the medium. The Cordus theory explains this as the fabric within a transparent object being denser, due to the proximity of the massy molecules, than the vacuum. Thus it is proposed that the fabric of the vacuum extends into and through all massy objects, and those objects densify it locally. Thus the Cordus theory provides a parsimonious way to reconcile the phenomenon of the speed of light varying with refractive index, and the in-vacuo behaviour of light.

A fourth contribution is that the Cordus theory reconciles many of the existing theories of light, despite their differences. Thus it accepts, albeit as approximations, the ideas from electromagnetic wave about light being an electromagnetic wave, that the vacuum is a medium, that the photon is a transient in the medium. It likewise accepts the quantum mechanics ideas of photons being individual items rather than waves. It also accepts, with caveats, the special relativity assumption that the speed of light is constant. The theory proposes that this is only approximately true because the mass density distribution of the Milky Way creates a uniform fabric density, hence a privileged location.

Falsifiable predictions

The Cordus theory is a conceptual work, built on a starting conjecture for the structure of matter. Falsifiable feature of the theory are:

1) That speed of light depends on fabric density. This feature differentiates the Cordus theory from all other VSL models.

2) Another falsifiable prediction is that the speed of light is variant to this day. By comparison, some of the other VSL models that are time-based propose that the speed of light is now invariant.

\section{Author Contributions}

All authors contributed to the creation of the underlying particule concept, development of the ideas, and editing of the paper. DP wrote the first draft of the paper.

\section{Conflict of interest statement}

The authors declare no funding sources external to their affiliations. The authors declare that there is no conflict of interests regarding the publication of this article. The research was conducted without personal financial benefit from any external funding body, nor did any such body influence the execution of the work or the decision to publish.

\section{References}

Abdo, A. A., Ackermann, M., Ajello, M., Asano, K., Atwood, W. B., Axelsson, M., ... \& Bastieri, D. (2009). A limit on the variation of the speed of light arising from quantum gravity effects. Nature, 462(7271), 331-334. http://dx.doi.org/10.1038/nature08574

Albrecht, A., \& Magueijo, J. (1999). Time varying speed of light as a solution to cosmological puzzles. Physical Review D, 59(4), 13. http://dx.doi.org/10.1103/PhysRevD.59.043516

Barrow, J. D., \& Magueijo, J. (1999). Solving the flatness and quasi-flatness problems in Brans-Dicke cosmologies with a varying light speed. Classical and Quantum Gravity, 16(4), 1435-1454. http://dx.doi.org/10.1088/0264-9381/16/4/030 
Bassett, B. A., Liberati, S., Molina-Paris, C., \& Visser, M. (2000). Geometrodynamics of variable-speed-of-light cosmologies. Physical Review D, 62(10), 18. http://dx.doi.org/10.1103/PhysRevD.62.103518

Berman, M. S. (2011). General relativity with variable speed of light and Pioneers anomaly. Astrophysics and Space Science, 336(2), 327-329. http://dx.doi.org/10.1007/s10509-011-0839-y

Bradley, J. (1729). An account of the new discovered motion of the fixed stars. Philosophical Transactions, 637-661.

Clayton, M. A., \& Moffat, J. W. (2003). Scale invariant spectrum from variable speed of light metric in a bimetric gravity theory. Journal of Cosmology and Astroparticle Physics, (7), 13.

de Broglie, L. (1925). Recherches sur la théorie des quanta (Researches on the quantum theory). Annales de Physique, 3(10). http://tel.archives-ouvertes.fr/docs/00/04/70/78/PDF/tel-00006807.pdf

Einstein, A. (1920). Relativity: The special and general theory. New York: Holt.

FIPS. (1993). Integration Definition for Function Modeling (IDEF0). Retrieved from http://www.itl.nist.gov/ fipspubs/idef02.doc

Harko, T., \& Mak, M. K. (1999). Particle creation in varying speed of light cosmological models. Classical and Quantum Gravity, 16(8), 2741-2752. http://dx.doi.org/10.1088/0264-9381/16/8/312

Herrmann, S., Senger A., Mohle K., Nagel M., Kovalchuk E. V., \& A., P. (2009). Rotating optical cavity experiment testing Lorentz invariance at the $10^{\wedge}\{-17\}$ level. Phys. Rev. D, 80(10), 105011. http://dx.doi.org/10.1103/PhysRevD.80.105011

Magueijo, J. (2000). Covariant and locally Lorentz-invariant varying speed of light theories. Physical Review D, 62(10), 15. http://dx.doi.org/10.1103/PhysRevD.62.103521

Magueijo, J. (2000). Covariant and locally Lorentz-invariant varying speed of light theories. Physical Review D, 62(10), 103521. Retrieved from http://link.aps.org/doi/10.1103/PhysRevD.62.103521

Magueijo, J. (2001). Stars and black holes in varying speed of light theories. Physical Review D, 63(4), 14. http://dx.doi.org/10.1103/PhysRevD.63.043502

Magueijo, J. (2003). New varying speed of light theories. Reports on Progress in Physics, 66(11), 2025-2068. http://dx.doi.org/10.1088/0034-4885/66/11/r04

Magueijo, J. (2008). DSR as an explanation of cosmological structure. Classical and Quantum Gravity, 25(20), 8. http://dx.doi.org/10.1088/0264-9381/25/20/202002

Michelson, A. A., \& Morley, E. W. (1887). On the Relative Motion of the Earth and the Luminiferous Ether. American Journal of Science, 34, 333-345.

Moffat, J. W. (2005). Variable speed of light cosmology and bimetric gravity: An alternative to standard inflation. International Journal of Modern Physics A, 20(6), 1155-1162. http://dx.doi.org/10.1142/s0217751x050 24031

Pons, D. J. (2015). Inner process of Photon emission and absorption. Applied Physics Research, 7(4), 14-26. http://dx.doi.org/10.5539/apr.v7n4p24

Pons, D. J. (2015). Internal structure of the photon (Image licence Creative Commons Attribution 4.0). Wikimedia Commons (Creative Commons Attribution 4.0 International license). Retrieved from https://commons. wikimedia.org/wiki/File:Internal_structure_of_the_photon.jpg

Pons, D. J., \& Pons, A. D. (2013). Outer boundary of the expanding cosmos: Discrete fields and implications for the holographic principle The Open Astronomy Journal, 6, 77-89. http://dx.doi.org/10.2174/18743811013 06010077

Pons, D. J., Pons, A. D., \& Pons, A. J. (2013a). Explanation of the Table of Nuclides: Qualitative nuclear mechanics from a NLHV design. Applied Physics Research 5(6), 145-174. http://dx.doi.org/10.5539/ apr.v5n6p145

Pons, D. J., Pons, A. D., \& Pons, A. J. (2013b). Synchronous interlocking of discrete forces: Strong force reconceptualised in a NLHV solution Applied Physics Research, 5(5), 107-126. http://dx.doi.org/10.5539/apr. v5n5107

Pons, D. J., Pons, A. D., \& Pons, A. J. (2013c). Time: An emergent property of matter. Applied Physics Research, 5(6), 23-47. http://dx.doi.org/10.5539/apr.v5n6p23 
Pons, D. J., Pons, A. D., \& Pons, A. J. (2014a). Asymmetrical genesis by remanufacture of antielectrons. Journal of Modern Physics, 5, 1980-1994. http://dx.doi.org/10.4236/jmp.2014.517193

Pons, D. J., Pons, A. D., \& Pons, A. J. (2014b). Differentiation of Matter and Antimatter by Hand: Internal and External Structures of the Electron and Antielectron. Physics Essays, 27, 26-35. http://dx.doi.org/10.4006/ 0836-1398-27.1.26

Pons, D. J., Pons, A. D., \& Pons, A. J. (2015a). Asymmetrical neutrino induced decay of nucleons Applied Physics Research, 7(2), 1-13. http://dx.doi.org/10.5539/apr.v7n2p1 or http://vixra.org/abs/1412.0279

Pons, D. J., Pons, A. D., \& Pons, A. J. (2015b). Hidden variable theory supports variability in decay rates of nuclides Applied Physics Research, 7(3), 18-29. http://dx.doi.org/10.5539/apr.v7n3p18

Pons, D. J., Pons, A. D., \& Pons, A. J. (2015c). Nuclear polymer explains the stability, instability, and non-existence of nuclides. Physics Research International, 2015(Article ID 651361), 1-19. http://dx.doi.org/10.1155/2015/651361

Pons, D. J., Pons, A. D., \& Pons, A. J. (2015d). Pair Production Explained in a Hidden Variable Theory Journal of Nuclear and Particle Physics, 5(3), 58-69. http://dx.doi.org/10.5923/j.jnpp.20150503.03

Pons, D. J., Pons, A. D., Pons, A. M., \& Pons, A. J. (2012). Wave-particle duality: A conceptual solution from the cordus conjecture. Physics Essays, 25(1), 132-140. http://dx.doi.org/10.4006/0836-1398-25.1.132

Racker, J., Sisterna, P., \& Vucetich, H. (2009). Thermodynamics in variable speed of light theories. Physical Review D, 80(8). http://dx.doi.org/10.1103/PhysRevD.80.083526

Schrödinger, E. (1926). An Undulatory Theory of the Mechanics of Atoms and Molecules. Physical Review, 28(6), 1049-1070. Retrieved from http://link.aps.org/doi/10.1103/PhysRev.28.1049

Shojaie, H. (2012). Variable speed of light cosmology as a solution to the Pioneer anomaly. Canadian Journal of Physics, 90(3), 229-234. http://dx.doi.org/10.1139/p2012-009

Singha, A. K., \& Debnath, U. (2007). Varying speed of light, modified chaplygin gas and accelerating universe. International Journal of Modern Physics D, 16(1), 117-122. http://dx.doi.org/10.1142/s0218271807009358

Sisterna, P. D. (2011). Variable speed of light theories: A thermodynamic analysis of planetary and white dwarf phenomena. International Journal of Modern Physics D, 20(5), 805-820. http://dx.doi.org/10.1142/s0218271 811019141

Szydlowski, M., \& Krawiec, A. (2003). Dynamical system approach to cosmological models with a varying speed of light. Physical Review D, 68(6), 14. http://dx.doi.org/10.1103/PhysRevD.68.063511

Unzicker, A. (2009). A look at the abandoned contributions to cosmology of Dirac, Sciama, and Dicke. Annalen Der Physik, 18(1), 57-70. http://dx.doi.org/10.1002/andp.200810335

Youm, D. (2001). Brane world cosmologies with varying speed of light. Physical Review D, 63(12), 125011. Retrieved from http://link.aps.org/doi/10.1103/PhysRevD.63.125011

\section{Copyrights}

Copyright for this article is retained by the author(s), with first publication rights granted to the journal.

This is an open-access article distributed under the terms and conditions of the Creative Commons Attribution license (http://creativecommons.org/licenses/by/3.0/). 